Tutolo $\mathrm{M}^{1}$, Bekhuis $\mathrm{Y}^{1}$, Everaerts W1ํ. Briganti $\mathrm{A}^{2}$, Joniau $\mathrm{S}^{1}$, Castagna $\mathrm{G}^{2}$, Ammirati $\mathrm{E}^{3}$, Zaffuto $\mathrm{E}^{2}$, De Ridder $\mathrm{D}^{1}$

1. Department of Urology, University Hospitals, KU Leuven, Belgium, 2. Urological Research Institute, IRCCS Ospedale San Raffaele, Division of Oncology/Unit of Urology, Milan, Italy, 3. Azienda Ospedaliera Universitaria, Città della Salute e della Scienza, Ospedale Molinette, Università di Torino, Turin, Italy

\title{
IS THE ICIQ-SF QUESTIONNAIRE RELIABLE IN A REAL-LIFE SETTING? RESULTS OF A PROSPECTIVE SINGLE-CENTER STUDY
}

Hypothesis / aims of study

The aim of the study is to assess functional outcomes, using validated questionnaires, in a large group of patients after robotassisted radical prostatectomy (RARP) in a single center. We also assessed the correlation between ICIQ-SF and EORTC QLQC30 results in order to evaluate the association between objective measurements and "return to normal activities" which represents the "real life" functional goal of this technique.

\section{Study design, materials and methods}

363 patients underwent RARP between September 2009 and August 31st 2016. All data were prospectively collected in a compulsory regional database (Flemish cancer registry). The database was accessed on August 2016. Pre- and post-operative ICIQ-SF and EORTC QLQ-C30 questionnaires were available for every patient. Minimal clinical important difference (MCID) values between pre and post-operative scores (defined as the smallest difference in score in the domain of interest which patients perceive as successful) were used to define a successful outcome after surgery. Paired T-test analyses were used to compare pre and post-operative results. Patients where then subdivided, according to the MSID, in two groups: patients with successful outcome and without successful outcome. Chi-squared test was used to investigate the relationship between ICIQ-SF and EORTC QLQ-C30 results.

Results

Mean follow up was 13.5 months (median11, range 1-43). Before surgery, $81 \%$ of patients reported a ICIQ-SF score of 0 . Using $M C I D=4,11$ points, this proportion decreased to $60 \%(N=227), p=0.26$. At baseline $45 \%$ of patients had Global health scores of $100(45 \%)$. After surgery this proportion was $52 \%$. MCID was $6,67,(p=0.00)$. Before surgery $76 \%$ and $88 \%$ of patients resulted to have 100 points in Physical and Role functioning respectively. After surgery this proportion decreased to $71.6 \%$ and $67 \%$ $(p=0.89,0.043)$ respectively. MCID resulted to be 6.4 and 9.1 points respectively. The results obtained for each EORTC QLQC30 domain were compared to the objective results of the ICIQ-SF. Satisfactory EORTC outcomes were not associated to satisfactory ICIQ-SF scores (all $p \leq 0.05$ ).

Interpretation of results and Concluding message

According to our knowledge, this is the first prospective, single center study comparing ICIQ-SF results and the real-life outcomes addressed with EORTC QLQ-C30, of a big cohort of patients submitted to RARP. We showed that quality of life is not only determined by objective continence outcomes and should be associated with more true-life questionnaires, in order to improve the knowledge and the treatment of this group of patients.

Disclosures

Funding: none Clinical Trial: No Subjects: HUMAN Ethics not Req'd: retrospective Helsinki: Yes Informed Consent: Yes 